\title{
Interpretation of ${ }^{41} \mathrm{Ca}$ data using compartmental modeling in post-menopausal women
}

\author{
Wang-Hee Lee, \\ Department of Agricultural and Biological Engineering, Purdue University, West Lafayette, IN \\ 47907-2093, USA \\ Meryl E. Wastney, \\ Metabolic Modeling Services Ltd, Blenheim, New Zealand \\ George S. Jackson, \\ PRIME Lab, Purdue University, West Lafayette, IN 47907-2036, USA \\ Berdine R. Martin, and \\ Department of Foods and Nutrition, Purdue University, 700 West State St, West Lafayette, IN \\ 47907-2059, USA

\section{Connie M. Weaver} \\ Department of Foods and Nutrition, Purdue University, 700 West State St, West Lafayette, IN \\ 47907-2059, USA \\ Connie M. Weaver: weavercm@purdue.edu
}

\section{Abstract}

Calcium-41 $\left(t_{1 / 2}=10^{5}\right.$ years $)$ can be used after a single dose to follow calcium metabolism over a subject's lifetime. The aims of this study were to expand a ${ }^{41} \mathrm{Ca}$ kinetic model and estimate bone resorption in women with stable bone loss, compare the rates with those calculated with classical isotope studies, and to use the model to simulate dynamic changes in urinary ${ }^{41} \mathrm{Ca}: \mathrm{Ca}$ ratios and bone balance for the design and interpretation of $41 \mathrm{Ca}$ studies. Forty-two women $>5$ years postmenopause were given ${ }^{41} \mathrm{Ca}$ intravenously. Bone mineral content and bone mineral density of total body were measured by dualenergy X-ray absorptiometry at the beginning of the study. Urine collections were made periodically for up to $\sim 5$ years while subjects were free living.

Urinary ${ }^{41} \mathrm{Ca}$ :Ca ratios were measured using accelerator mass spectrometry. The isotope data were analyzed by compartmental modeling. Four compartments were necessary to fit the urinary tracer data and total bone calcium. The final model included pathways for absorption, distribution, urinary excretion, and endogenous excretion and was used to calculate rates of bone turnover. Estimates of bone resorption in a subset of the women $(n=13)$, studied previously in a 3-week balance and full kinetic study with ${ }^{45} \mathrm{Ca}$, agreed with those using ${ }^{41} \mathrm{Ca}$ methodology. Thus, rates of bone resorption can be estimated from $41 \mathrm{Ca}$ urinary data in stable postmenopausal women. The model was used to simulate dynamic changes in urinary 41Ca:Ca ratios and bone balance, as a result of interventions that perturb calcium metabolism to aid in study design and interpretation. 


\section{Keywords \\ ${ }^{41} \mathrm{Ca}$; Compartmental modeling; Bone resorption; Bone balance; Dynamic simulation}

\section{Introduction}

Qualitative changes in bone turnover that result in gain (or loss) of bone can be currently assessed using biomarkers [1, 2]. Dual-energy X-ray absorptiometry provides a quantitative measure of bone gain (loss) after the changes have occurred [3]. It would be useful to have an alternative method for determining more rapidly the rate of bone loss (or gain) following an intervention. A recent approach uses ${ }^{41} \mathrm{Ca}$, a radioisotope with a half-life $\left(t_{1 / 2}=\sim 10^{5}\right.$ years), which is long compared to the lifespan of a biological entity, and thus behaves like a stable isotope. The excretion of ${ }^{41} \mathrm{Ca}$ can be determined in single urine samples for years after a single dose because of the sensitivity of measurement by accelerator mass spectrometry (AMS) and the lack of abundance of ${ }^{41} \mathrm{Ca}$ (natural abundance $<10^{-15}$ ) in the environment [4]. Denk et al. administered ${ }^{41} \mathrm{Ca}$ to postmenopausal women and analyzed urine data with a three-compartment model [5]. Changes in ${ }^{41} \mathrm{Ca}$ urinary excretion after bisphosphonate intervention paralleled changes in biochemical markers and bone mineral density (BMD) [6], although they did not calculate rates of bone turnover.

While the potential for ${ }^{41} \mathrm{Ca}$ for determining changes in bone turnover has been demonstrated, interpretation of the data still has some unanswered questions, namely, what changes in $\mathrm{Ca}$ metabolism alter the ${ }^{41} \mathrm{Ca}$ :Ca ratio? Does a change in ratio always correspond to a change in bone balance? Do interventions at different times after dosing cause the same response in ratio? How large a change in metabolism is required for it to be detectable in the ratio? How long a recovery period (i.e., for bone balance to return to pre-intervention level) is required after stopping a treatment (e.g., as with bisphosphonate)? These questions can be addressed through dynamic modeling.

The purpose of this study was threefold: (1) to expand a kinetic model [5] for ${ }^{41} \mathrm{Ca}$ in postmenopausal women who are beyond the rapid bone loss phase (i.e., have stable bone loss) [7] for calculating bone turnover rate, (2) to compare the values calculated for bone resorption with those calculated from previous isotope studies in a sub-group of the subjects, and (3) to use the model to simulate dynamic changes in urinary ${ }^{41} \mathrm{Ca}$ :Ca ratios and bone balance for the design and interpretation of ${ }^{41} \mathrm{Ca}$ studies.

\section{Materials and methods}

\section{Subjects}

Forty-two post-menopausal women aged 37 to 74 years were studied (Table 1). Subjects were excluded if they were less than 4 years post-menopausal to ensure that bone loss is in stable state. Also, subjects that used estrogen hormone replacement therapy, antiresorptive drugs, drugs for the treatment of bone disease, thiazide diuretics, thyroid medication, corticosteroids, non-prescriptive drugs, or consumed isoflavones were excluded since the following study will explore the effect of isoflavone-like treatment on bone metabolism. 
Potential subjects were also excluded if their medical history included mal-absorptive disorders, bone, liver, or kidney disease, hormonal abnormalities, cancer, or soy allergies. Serum follicle-stimulating hormone and luteinizing hormone were measured at screening to verify post-menopausal status.

The Human Institutional Review Boards at Purdue University, Indiana University-Purdue University Indianapolis, and Clarian IRB approved the protocol in this study as part of larger intervention studies.

\section{Study design}

Subjects participated in several studies including two previously reported [8, 9]. However, only the baseline or pretreatment data while the subjects were free-living were used here. Due to the long-term nature of the studies, the subjects for any one study were not studied simultaneously, but at intervals. Bone mineral content (BMC) and BMD of the lumbar spine, proximal femur, and total body were measured by dual-energy X-ray absorptiometry (Lunar DPX IQ or Prodigy, Madison, WI, USA) at the beginning of the study. Subjects were provided 500-648 mg calcium per day and 400-500 IU/day of vitamin D (Viactive, McNeil Nutritionals, LLC, Ft. Washington, PA, USA; Rexall Sundown, Inc., Boca Raton, FL, USA; Geritol, Glaxo Smith Kline, England) throughout the study period.

They were dosed with 50 to $1,000 \mathrm{nCi}{ }^{41} \mathrm{Ca}$ (Argonne National Laboratories) in sterile saline by a single intravenous injection (six subjects received it in three divided doses). The larger doses $(1 \mu \mathrm{Ci})$ were administered initially, but smaller doses were administered in later studies as we learned that these were sufficient for detection. Both the size and administration schedule of the dose were modeled in the data analysis.

\section{Sample collection}

Urine samples (at least $500 \mathrm{ml}$ ) were collected beginning with the second void of the day every 10-20 days for 167-1,808 days after dosing. The wide time range in the baseline is indicative of multiple studies taking place over several years. Initially, urine collections were requested until the approximate total calcium excreted for each individual was established for determining the volume required for $41 \mathrm{Ca}$ analysis. Then, individual volume collections that provided the minimum calcium for analysis were then requested (e.g., 500 $\mathrm{ml}$ or 1 1) up to a maximum of a $24-\mathrm{h}$ volume. The volume collected depended on the urinary calcium concentration of the particular subject. For approximately one out of every eight woman, it was determined that a 24-h urine collection was required to provide the 15$20 \mathrm{mg}$ of $\mathrm{CaF}_{2}$ necessary to fill the AMS sample holder when mixed with silver. We lose some calcium during the processing, and though measurements can be made with less material, the focusing properties of our source are such that the highest output is obtained when our sample well is full (1.2 mm diameter, and $2 \mathrm{~mm}$ deep).

\section{Sample analysis}

Urine samples were collected in acid-washed containers, and the volume of each urine collection was measured. Approximately $10 \mathrm{ml}$ of ammonium hydroxide was added to every liter of urine and mixed to ensure that the $\mathrm{pH}$ was at least 10 . Then, $50 \mathrm{ml}$ of saturated 
ammonium oxalate was added and mixed to separate the calcium by precipitation as calcium oxalate $\left(\mathrm{CaC}_{2} \mathrm{O}_{4}\right)$ by letting the sample stand overnight. The supernatant was decanted, leaving the precipitate and some urine, which were then filtered. The precipitate was dissolved in $50 \mathrm{ml}$ of approximately $0.5 \mathrm{M} \mathrm{HNO}_{3}$ solution and chromatographically purified by cation exchange using Bio-Rad AG 50W-X8 resin (Bio-Rad, Hercules, CA, USA). Calcium fluoride $\left(\mathrm{CaF}_{2}\right)$ was precipitated with hydrofluoric acid, washed, dried in a vacuum oven, loaded into aluminum sample holders, and inserted into the ion source of the AMS to obtain the ${ }^{41} \mathrm{Ca}$ :Ca ratio of the samples.

At the Purdue Rare Isotope Measurement Laboratory (PRIME Lab), the samples were measured during six runs that took place between 2000 and 2009. PRIME Lab is an NSFsupported AMS facility that also makes measurements for the life sciences. The AMS at PRIME Lab uses an upgraded FN (nominal $8 \mathrm{MV}$ ) tandem accelerator with a cesium sputter negative ion source, a 150-kV injector, and beam lines containing three large sector magnets. The detector is a gas-filled ionization detector that can discriminate between nuclear isobars. The AMS is approximately $60 \mathrm{~m}$ from ion source to detector. The mean value of each ${ }^{41} \mathrm{Ca}$ :Ca isotope ratio was determined by normalizing the measured value against the measured values of two standards, one measured before and one measured after each unknown. The standards used had a ${ }^{41} \mathrm{Ca}$ :Ca ratio of either $3.7 \times 10^{-10}$ or $2 \times 10^{-9}$. Each sample was measured multiple times, and the uncertainty was calculated from counting statistics and systematic errors [10]. In brief, the uncertainty from counting statistics is $\sigma=1 \sqrt{N}$, where $N$ is the number of counts in the detector. Systematic errors most likely arise from small imperfections as the ion beam is jockeyed around the beam line. They were accounted for by calculating the standard deviation of the ratio each time a sample was measured. The total number of ${ }^{41} \mathrm{Ca}$ measurements was about 630, and the average number of $41 \mathrm{Ca}$ measurements per subject was 15 with a range from 6 to 52 . The typical precision of a measurement was 5\% with a range from $2 \%$ to $7 \%$. If a sample had precision over $10 \%$, that indicates that something had gone awry with the AMS measurement, and the ratio was disregarded. The background due to chemical processing was regularly checked by running blanks over our chromatography columns, one a calcium solution and the other a urine specimen. The Purdue ion source generates $\mathrm{CaF}_{3}{ }^{-}$ion currents of 50-500 nA with the corresponding ${ }^{41} \mathrm{Ca}^{8+}$ ion detection rates of about $3 \mathrm{~Hz}$ for a sample ${ }^{41} \mathrm{Ca}$ enrichment of about $5 \times 10^{-10}$ of total calcium. A background of $5 \times 10^{-13}$ is routinely achievable with samples collected prior to tracer administration. Background subtraction was performed on all unknowns as part of our analysis procedure but was unnecessary for most unknowns.

Total calcium in 24-h urine was measured in 17 subjects using atomic absorption spectrophotometry as previously described [11].

\section{Kinetic modeling in steady state}

Kinetic calculations during the baseline period assumed steady-state (i.e., that pool sizes were constant), so the rate of bone resorption equaled bone formation. A compartmental model was fitted to the urinary ${ }^{41} \mathrm{Ca}: \mathrm{Ca}$ data, measures of total bone calcium, and where available, daily urine $\mathrm{Ca}$, for each subject using WinSAAM [12]. The dosing regimen (single or multiple i.v. doses of tracer) was simulated. The model was fitted using least 
squares procedures [12]. Modeling notation was $L(i, j)$, for daily factional transfer from compartment $j$ into compartment $i, M(i)$ for compartmental mass in milligrams, and $R(i, j)$ for daily mass transfer from compartment $j$ into compartment $i$ (i.e., $R(i, j)=L(i, j) \times M(j)$ (milligrams per day)). The mass of compartment 1 was calculated as the product of initial volume of distribution and plasma $\mathrm{Ca}$ concentration. Compartment turnover time (day) was calculated as the inverse of the sum of all the $L(i, j)$ out of a compartment. Compartment mass, rates of calcium absorption, bone resorption (or formation), urine excretion, endogenous excretion, the diet necessary to maintain bone mass, the initial volume of distribution, and total bone calcium were calculated by the model. The initial volume of distribution was calculated based on i.v. tracer distribution (i.e., $y$-intersection of i.v. tracer). The dietary intake of calcium necessary to maintain bone mass was determined as an input to the model.

\section{Dynamic simulations}

To simulate calcium dynamics, when pool sizes change over time and bone formation and bone resorption rates differ (as would occur after a treatment or diet change), we modeled tracee $\left({ }^{40} \mathrm{Ca}\right)$ and tracer simultaneously, using the population values for the parameters. In detail, we set up a duplicate model for tracee that had the same structure as the tracer model. The initial condition for each compartment in the tracee model was set to the steady-state mass of calcium as calculated by the tracer model. The ${ }^{41} \mathrm{Ca}$ :Ca ratio was calculated as the tracer in compartment 1 divided by the amount of calcium in the analogous compartment in the tracee model, since we assume that urinary and serum ratios are equivalent [13]. We simulated both models for $\sim 250$ days after administration of an i.v. dose (baseline period) and then simulated a treatment (or intervention) by introducing changes (in the range of 50$200 \mathrm{mg} /$ day) in various pathways that would have an effect on bone balance (defined as the difference between bone deposition and bone resorption). We calculated the effect of the change on the ${ }^{41} \mathrm{Ca}$ :Ca ratio and on bone balance since this determines bone mass.

\section{Statistical analysis}

Paired $t$ test was used to compare the values of the bone resorption rate and urinary calcium excretion between this study and a previously reported 3-week balance study using ${ }^{45} \mathrm{Ca}$ that shared a subset of subjects [14]. SAS statistical software package (version 9.1.3, SAS Institute Inc., Cary, NC, USA) was used for statistical analysis.

\section{Results}

\section{Kinetic modeling}

A three-compartment model was initially employed to fit the ${ }^{41} \mathrm{Ca}$ data as in a previous study [5], but the model was expanded by including pathways for absorption, excretion into urine, and endogenous excretion. Modeling assumptions were based on a combined kinetic and balance study in post-menopausal women [14] that absorption was $20 \%$ of dietary intake, endogenous excretion was $150 \mathrm{mg} /$ day and, if not measured, urinary $\mathrm{Ca}$ excretion was $150 \mathrm{mg} /$ day. For subjects whose data were collected for $<300$ days, the value for $L(2,3)$, which represents the fraction of Ca moving from compartment 3 to compartment 2 , was set to the average value $\left(2.5 \times 10^{-3} /\right.$ day $)$ calculated for subjects with data obtained for $>300$ 
days. While the three-compartment model fitted the urinary ratio data, it underestimated the mass of calcium in the body (estimated as 38\% of BMC [15]) calculated as the sum of calcium mass in the three compartments. Thus, it was necessary to add a fourth compartment to the model (Fig. 1). We chose to define the pool based on knowledge of two forms of bone, trabecular and cortical, known to turnover at different rates. We refer to compartment 3 , the faster turning over bone compartment, as "trabecular" and compartment 4 as "cortical" bone. The ratio of Ca in cortical vs. trabecular bone is considered to be 80:20 [16], and so the fractional transfer into compartment 4 was set so that its mass was four times that of compartment 3. Cortical bone turnover is reported to be 4.4\%/year [17], which is a turnover time of over 20 years. As it was too slow to be determined from our data, release from compartment $4, L(2,4)$, was set using this value $\left(10^{-4} /\right.$ day $)$. Whole body calcium data were fitted by the sum of the mass of compartments $1,2,3$, and 4 . Fits of the four-compartment model to data are shown in Fig. 2. A plot of the distribution of tracer remaining in the body shows that from 250 until $\sim 1,800$ days, most was in compartment 3 , the "trabecular" bone compartment; thereafter, tracer was predominantly in compartment 4 (Fig. 3).

The initial volume of distribution was calculated as $53 \pm 23 \mathrm{~L}$. The average transfer coefficients (Fig. 1a) were used to determine turnover times of $11 \pm 5,118 \pm 92,396 \pm 187$, and 10,000 days for compartments 1, 2, 3, and 4, respectively. The pool sizes in gram $\mathrm{Ca}$ and mass transfers in milligrams per day (calculated from averaging $R(i, j)$ across all subjects, Fig. 1b) show the higher rate of resorption from "trabecular" vs. "cortical bone" (501 vs. 69 $\mathrm{mg} /$ day). Because we assume steady state, the rates of bone resorption (the sum of $\mathrm{Ca}$ transport from compartment 3 to compartment 2 and out of compartment 4) and bone formation (transport from compartment 2 to compartments 3 and 4) were the same (Fig. 1b). Calculated whole body $\mathrm{Ca}$ was $945 \pm 171 \mathrm{~g}$. The percent distribution of $\mathrm{Ca}$ from each compartment (Fig. 1c) shows, for example, that of calcium entering compartment 1,50\% will go to compartment 2 , while $25 \%$ is excreted in urine and $25 \%$ in feces.

For a subset of the women $(n=13)$, bone resorption rates calculated by the model from only urine ${ }^{41} \mathrm{Ca}$ data were compared to the rate calculated previously using ${ }^{45} \mathrm{Ca}$ sampled in multiple tissues (serum, urine, and feces) following oral and i.v. tracer administration [14]. For these subjects, bone resorption rates calculated from the ${ }^{41} \mathrm{Ca}$ and the $45 \mathrm{Ca}$ data were not statistically different $(p=0.21$; Table 2$)$.

\section{Dynamic simulations of ${ }^{41} \mathrm{Ca}: \mathrm{Ca}$ ratio}

To aid in experimental design and interpretation of ${ }^{41} \mathrm{Ca}$ data, we simulated the effect of changes in calcium metabolism (representing an intervention or therapy) on predicted urinary ${ }^{41} \mathrm{Ca}$ :Ca ratio and bone balance. We simulated a $1-\mu \mathrm{Ci}$ i.v. dose of ${ }^{41} \mathrm{Ca}$ for a baseline period of 250 days and then changed some parameter values. Two assumptions were made: changes we introduced to pathways were the only changes that occurred in the system (i.e., there were no other homeostatic responses), and because plasma calcium is tightly controlled, the mass of tracee in the first pool (containing plasma) remained constant after any parameter change. So, if we perturbed a parameter that increased calcium entering the first compartment, we also increased a loss pathway to keep that pool constant. For 
example, if we increased calcium entry into plasma from bone resorption, we also increased urinary loss or, alternatively, decreased calcium absorption. In this way, pairs (or triplets) of parameters were altered in each simulation; 15 combinations were tested (Tables 3 and 4). The ${ }^{41} \mathrm{Ca}$ :Ca ratio and bone balance were calculated at 500 days post-dose (i.e., after 250 days of intervention). The change in bone balance was dynamic or changing over time. If an intervention was continued (beyond 250 days), we found that it took more than 10 years for balance to return to pre-intervention values (Fig. 4).

Results of each simulation are expressed in two ways, as percent change, so that the degree of change in the pathway can be compared to the resulting change in ratio (Table 3) and on an absolute basis (Table 4). The changes refer to the pathways and transport rates in Fig. 1b. Results for the specific questions posed are as follows:

1. To answer what changes in Ca metabolism alter the urinary ${ }^{41} \mathrm{Ca}$ :Ca ratio, all the simulations tested showed a change in the ${ }^{41} \mathrm{Ca}$ :Ca ratio (Tables 3 and 4).

2. Does a change in ratio always correspond to a change in bone balance? The change in ratio corresponded inversely with bone balance except for two cases, when a decrease (or increase) in absorbed calcium was matched by a decrease (or increase) in urine excretion (simulations 1 and 5, Table 4). In all other cases, increased bone balance was associated with decreased ${ }^{41} \mathrm{Ca}$ :Ca ratio and vice versa.

3. Do interventions at different times after dosing cause the same response in ratio? Simulation results were consistent with respect to changes in ratio and bone balance when the intervention was introduced at either 250 or 2,000 days post-dose (Table 5).

4. To determine how small a change in metabolism can be detected in the ratio, we introduced interventions of varying size in absorption, excretion, and bone formation at 250 days post-dose and simulated for 50 days. As expected, the simulations showed that the change in the ${ }^{41} \mathrm{Ca}$ :Ca ratio became larger as the magnitude of the intervention increased. Specifically, the model predicted that a change of less than $15 \mathrm{mg} /$ day in bone balance changed the ratio about $5 \%$, whereas a $30-\mathrm{mg} /$ day change in bone balance caused a $\sim 10 \%$ change in the ratio (data not shown).

5. To address how long a recovery period is required following an intervention for bone balance to return to the pre-intervention level, we simulated the model for baseline (250 days), intervention (50 days), and variable recovery periods, after model parameter values were reset to baseline values. The simulation showed that recovery of bone balance was dependent upon the magnitude of the intervention: when bone balance was perturbed by about $50 \mathrm{mg} /$ day, a 50-day recovery time returns bone balance within $10 \mathrm{mg} /$ day of its pre-intervention value, but more than 200 days of recovery is required to return bone balance to its original value. 


\section{Discussion}

By extending a previous model [5] to fit urinary ${ }^{41} \mathrm{Ca}$ data obtained over $>6$ months and calcium mass in bone, we estimated bone resorption rates for stable post-menopausal women. Because this tracer can be monitored for the lifetime of a subject after a single dose, potential uses for ${ }^{41} \mathrm{Ca}$ include monitoring changes in a subject's bone metabolism due to disease or therapy. In these situations, metabolism would not be in steady state, and so we used the model to perform dynamic simulations that may help in the implementation and analysis of data in ${ }^{41} \mathrm{Ca}$ studies with interventions.

We made several assumptions in expanding the model proposed by Denk et al. [5]. Two of these (absorption of calcium was $20 \%$ and rates of endogenous excretion of $150 \mathrm{mg} /$ day) used the mean values from a study in a population of post-menopausal women [14]. Denk et al. [5] assumed 50\% absorption as they administered their tracer orally. As we administered the dose i.v., we only assumed absorption of dietary calcium. We assumed steady state for the baseline period (but used a dynamic model for interventions). Bone loss 5 years after menopause is reported to be 1\%/year [18, 19]. For whole body Ca of $945 \mathrm{~g}$, this represents $26 \mathrm{mg} /$ day. We found that a $10 \%$ change in ${ }^{41} \mathrm{Ca}$ :Ca ratio is equivalent to about $30 \mathrm{mg} / \mathrm{day}$ in resorption (and with further simulation using the dynamic model, $26 \mathrm{mg} / \mathrm{day}$ represents a $7 \%$ difference in ratio). This is within the error of sample measurement of $2-7 \%$. We conclude that the $1 \%$ loss/year is a relatively small amount daily and within experimental error, supporting our use of steady state for the baseline period.

The results for resorption determined from ${ }^{41} \mathrm{Ca}$ data agreed with those from a study in one subject that included both oral and i.v. stable isotope tracer administration [20] and, as described in this paper, with 13 subjects that had blood sampling and complete collection of urine and feces for balance measurements [14]. This agreement suggests that bone resorption can be estimated using the less invasive ${ }^{41} \mathrm{Ca}$ methodology, which means that after tracer administration, only single urine voids are required, not blood draws.

Furthermore, Freeman et al. [21] reported there was no diurnal variation in the ${ }^{41} \mathrm{Ca}$ signal.

We previously used a statistical approach to evaluate changes in ${ }^{41} \mathrm{Ca}$ [9]. The current approach provides more quantitative information about calcium metabolism. Compared to the three-compartment model for 41Ca data published by Denk et al. [5], we found that most parameter values, including volume of distribution, transfer coefficients, and compartment turnover times, agreed. The turnover time of compartment 2 was slower, but quite variable, in our study (118 \pm 92 days) compared to Denk et al. (28 days) and may be related to our low sampling density during the first 50 days, when compartment 2 peaks [5]. Our estimates of data fitting were similar; the relative residual from our model was $0.8 \pm 10 \%$ similar to that reported by Denk et al. $(0 \pm 7 \%)$. The slightly larger residual may be due to our larger sample size and longer sampling time.

It should be noted that the compartments in the model based on the 3-week study [14] differ to those in the current study and that it is not possible to directly compare the parameter values. Specifically, the combined mass of all three compartments of the 3-week model $(6 \mathrm{~g})$ is comparable to only the first compartment ( $5 \mathrm{~g}$, Fig. $1 \mathrm{~b}$ ) of the ${ }^{41} \mathrm{Ca}$ model. The two 
models (from 3-week studies and over several years) could be combined into a sixcompartment model, but this comprehensive model would only be useful when data collection spanned from hours to years.

We hope that the design of experimental studies will be improved by our dynamic simulations of the relationship of ${ }^{41} \mathrm{Ca}$ :Ca to various calcium interventions and to bone balance. Following changes in calcium metabolic pathways, we predict that increased bone balance reduces the urinary ${ }^{41} \mathrm{Ca}: \mathrm{Ca}$ ratio, and conversely, decreased bone balance increases ${ }^{41} \mathrm{Ca}$ :Ca ratios. However, the ${ }^{41} \mathrm{Ca}$ :Ca ratio would change independent of bone balance if all the absorbed calcium is assumed to be excreted via urine. This would be unlikely because only $25 \%$ of calcium entering the first compartment (by absorption or exchange) was excreted in urine, although an intervention such as citrate which facilitates urine excretion might create this scenario. We note that the changes related to increased urinary excretion also apply to changes in endogenous fecal excretion. That is, because the same fraction of compartment 1 transfers to feces by endogenous excretion as to urine (25\%), any changes in endogenous excretion can show the same effect on the ratio and bone balance as the changes in urine excretion. Therefore, changes in the urinary ${ }^{41} \mathrm{Ca}$ :Ca ratio appear to reflect the changes in bone balance under normal physiological conditions.

The model simulation suggests that the time of intervention beyond 250 days post-dose does not significantly affect the calculated bone balance and ratio. Rather, the size and site of the intervention has a larger impact on the ratio. In a subset of 11 subjects from this study, in a blinded, randomized order, cross-over trial (50 days intervention and recovery periods with urine collections every 10 days), we observed a significant change in ratio of 5\% [9]. That is, with a small sample size $(n=11)$, ratio changes $\geq 5 \%$ were required for significance. The model predicts that a $10 \%$ change in the ratio corresponds to a change in bone balance of about $30 \mathrm{mg} /$ day. Either, perturbations in the ${ }^{41} \mathrm{Ca}$ :Ca ratio larger than $5 \%$ (which corresponds to changes in bone balance of over $15 \mathrm{mg} /$ day) or a larger sample size may be required to achieve statistically significant differences in intervention studies.

The duration of recovery needed between interventions is dependent on the magnitude of effect of an intervention, i.e., a longer recovery time is necessary following large changes in the urinary ${ }^{41} \mathrm{Ca}$ :Ca ratio. The model predicts that for a $15-\mathrm{mg} /$ day change in bone balance, which produces a significant change in ${ }^{41} \mathrm{Ca}$ :Ca ratio, a 50-day recovery period is sufficient. However, for a 50-mg/day change in bone balance, a 50-day duration is sufficient for recovering bone balance to within $10 \mathrm{mg} /$ day, which does not significantly impact the ${ }^{41} \mathrm{Ca}$ :Ca ratio, but more than 200 days are required for complete recovery. This suggests that if multiple treatments are being assessed, it is important to compare a treatment period with the recovery periods that immediately bracket the treatment period in time as a large change in balance will require a longer recovery time.

Bone balance, or the rate of deposition minus the rate of resorption, is the main factor causing changes in ${ }^{41} \mathrm{Ca}$ :Ca ratio, as most tracer is in the bone compartments by the time of an intervention. The ratio would not change when the amount of calcium absorbed increases, but only under the non-physiological condition of all this additional calcium being excreted. Therefore, the ${ }^{41} \mathrm{Ca}$ :Ca ratio can be used to follow changes in bone metabolism as a proxy of 
bone balance. Future experiments are required to verify this predicted behavior, for example, measuring the urinary ${ }^{41} \mathrm{Ca}: \mathrm{Ca}$ ratio and comparing to short-term changes in calcium balance or long-term changes in bone calcium content in humans in response to various treatments that alter bone balance.

\section{Conclusion}

We developed a four-compartment model for ${ }^{41} \mathrm{Ca}$ kinetics in post-menopausal women by fitting urinary ${ }^{41} \mathrm{Ca}$ and total bone calcium data. The calculated results for bone resorption agreed with previous studies using $45 \mathrm{Ca}$ and sampling of multiple tissues. Therefore, we can conclude that the proposed model can be used when there is stable bone loss to estimate bone resorption using ${ }^{41} \mathrm{Ca}$ measurements in urine. In addition, model simulations confirm that changes in urinary ${ }^{41} \mathrm{Ca}: \mathrm{Ca}$ ratio can capture changes in bone metabolism as a proxy for bone balance.

\section{Acknowledgments}

This study is funded by Purdue University, University of Alabama Botanical Center for Age Related Diseases, and National Institutes of Health (NIH) grants P50 AT00477.

\section{References}

1. Looker AC, Bauer DC, Chesnut CH 3rd, Gundberg CM, Hochberg MC, Klee G, Kleerekoper M, Watts NB, Bell NH. Clinical use of biochemical markers of bone remodeling: current status and future directions. Osteoporos Int. 2000; 11(6):467-480. [PubMed: 10982161]

2. Leeming DJ, Alexandersen P, Karsdal MA, Qvist P, Schaller S, Tanko LB. An update on biomarkers of bone turnover and their utility in biomedical research and clinical practice. Eur J Clin Pharmacol. 2006; 62(10):781-792. [PubMed: 16912870]

3. Hui SL, Zhou L, Evans R, Slemenda CW, Peacock M, Weaver CM, McClintock C, Johnston CC Jr. Rates of growth and loss of bone mineral in the spine and femoral neck in white females. Osteoporos Int. 1999; 9(3):200-205. [PubMed: 10450407]

4. Jackson GS, Weaver C, Elmore D. Use of accelerator mass spectrometry for studies in nutrition. Nutr Res Rev. 2001; 14(2):317-334. [PubMed: 19087429]

5. Denk E, Hillegonds D, Vogel J, Synal A, Geppert C, Wendt K, Fattinger K, Hennessy C, Berglund M, Hurrell RF, Walczyk T. Labeling the human skeleton with ${ }^{41} \mathrm{Ca}$ to assess changes in bone calcium metabolism. Anal Bioanal Chem. 2006; 386(6):1587-1602. [PubMed: 17033771]

6. Denk E, Hillegonds D, Hurrell RF, Vogel J, Fattinger K, Hauselmann HJ, Kraenzlin M, Walczyk T. Evaluation of ${ }^{41}$ calcium as a new approach to assess changes in bone metabolism: effect of a bisphosphonate intervention in postmenopausal women with low bone mass. J Bone Miner Res. 2007; 22(10):1518-1525. [PubMed: 17576167]

7. Recker R, Lappe J, Davies K, Heaney R. Characterization of perimenopausal bone loss: a prospective study. J Bone Miner Res. 2000; 15(10):1965-1973. [PubMed: 11028449]

8. Cheong JMK, Martin BR, Jackson GS, Elmore D, McCabe GP, Nolan JR, Barnes S, Peacock M, Weaver CM. Soy isoflavones do not affect bone resorption in postmenopausal women: a doseresponse study using a novel approach with ${ }^{41} \mathrm{Ca}$. J Clin Endocrinol Metab. 2007; 92(2):577-582. [PubMed: 17148567]

9. Weaver CM, Martin BR, Jackson GS, McCabe GP, Nolan JR, McCabe LD, Barnes S, Reinwald S, Boris ME, Peacock M. Antiresorptive effects of phytoestrogen supplements compared with estradiol or risedronate in postmenopausal women using ${ }^{41} \mathrm{Ca}$ methodology. $\mathrm{J}$ Clin Endocrinol Metab. 2009; 94(10):3798-3805. [PubMed: 19584189]

10. Elmore D, Conard N, Kubik PW, Fabryka-Martin J. Computer controlled isotope ratio measurements and data analysis. Nucl Instrum Methods Phys Res B. 1984; 5(2):233-237. 
11. Wastney ME, Martin BR, Peacock M, Smith D, Jiang XY, Jackman LA, Weaver CM. Changes in calcium kinetics in adolescent girls induced by high calcium intake. J Clin Endocrinol Metab. 2000; 85(12):4470-4475. [PubMed: 11134095]

12. Wastney, ME.; Patterson, BH.; Linares, OA.; Greif, PC.; Boston, RC. Investigating biological systems using modeling: strategies and software. Academic Press; San Diego: 1999.

13. Neer R, Berman M, Fisher L, Rosenberg LE. Multi-compartmental analysis of calcium kinetics in normal adult males. J Clin Invest. 1967; 46(8):1364-1379. [PubMed: 16695925]

14. Spence LA, Lipscomb ER, Cadogan J, Martin B, Wastney ME, Peacock M, Weaver CM. The effect of soy protein and soy isoflavones on calcium metabolism in postmenopausal women: a randomized crossover study. Am J Clin Nutr. 2005; 81(4):916-922. [PubMed: 15817872]

15. Cooper AR, Forbes RM, Mitchell HH. Further studies on the gross composition and mineral elements of the adult human body. J Biol Chem. 1956; 223(2):969-975. [PubMed: 13385244]

16. O'Flaherty EJ. A physiologically based kinetic model for lead in children and adults. Environ Health Perspect. 1998; 106(Suppl 6):1495-1503. [PubMed: 9860908]

17. O'Flaherty EJ. Modeling normal aging bone loss, with consideration of bone loss in osteoporosis. Toxicol Sci. 2000; 55(1):171-188. [PubMed: 10788572]

18. Dawson-Hughes B. Calcium and vitamin D nutritional needs of elderly women. J Nutr. 1996; 126(4 Suppl):1165S-1167S. [PubMed: 8642451]

19. McClung MR, Lewiecki EM, Cohen SB, Bolognese MA, Woodson GC, Moffett AH, Peacock M, Miller PD, Lederman SN, Chesnut CH, Lain D, Kivitz AJ, Holloway DL, Zhang C, Peterson MC, Bekker PJ. Denosumab in postmenopausal women with low bone mineral density. N Engl J Med. 2006; 354(8):821-831. [PubMed: 16495394]

20. Freeman SPHT, King JC, Vieira NE, Woodhouse LR, Yergey AL. Human calcium metabolism including bone resorption measured with ${ }^{41}$ Ca tracer. Nucl Instrum Methods Phys Res B. 1997; 123:266-270.

21. Freeman SPHT, Beck B, Bierman J, Caffee MW, Heaney RP, Holloway L, Marcus R, Southon JR, Vogel JS. The study of skeletal calcium metabolism with ${ }^{41} \mathrm{Ca}$ and ${ }^{45} \mathrm{Ca}$. Nucl Instrum Methods Phys Res B. 2000; 172(1-4):930-933. 

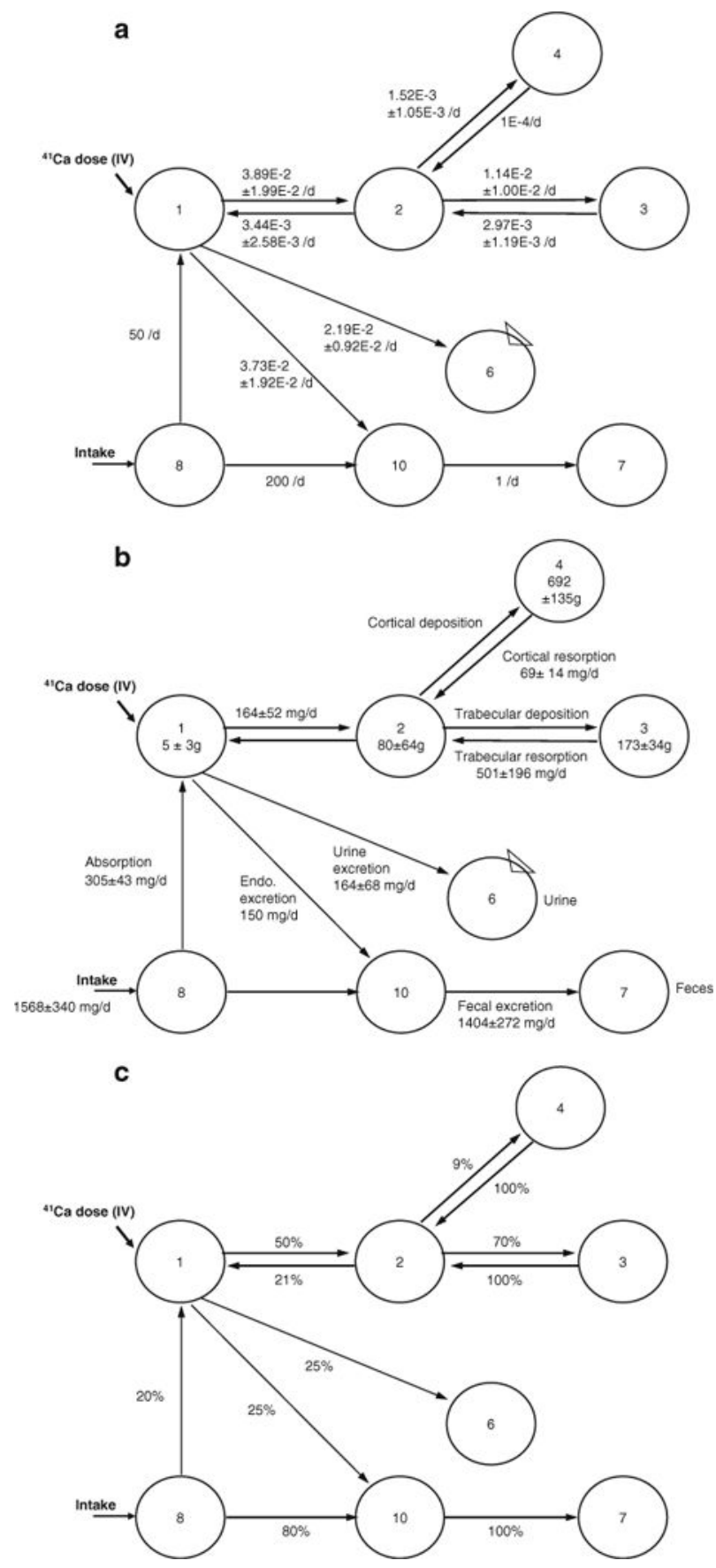

Fig. 1.

Compartmental model for calcium metabolism, with pools numbered arbitrarily with values for a fractional transfers $(L(i, j)$, into compartment $i$ from compartment $j)$, mean $\pm \operatorname{SD}(n=42)$, b pool size and mass transfer, and $\mathbf{c}$ percent distribution of Ca from the compartments where the arrows start. $L(6,1)$ was the average for subjects where urinary excretion was measured $(n=17)$. Absorption $[L(1,8) /(L(1,8)+L(10,8)]$ was fixed at $20 \%$, and endogenous excretion, $L(10,1)$, was based on a rate of $150 \mathrm{mg} /$ day [14]. Compartment 4 was necessary to fit both 
urinary tracer and bone calcium data, and $L(2,4)$ was fixed based on cortical bone turnover of $4.4 \% / y e a r[16,17]$ 


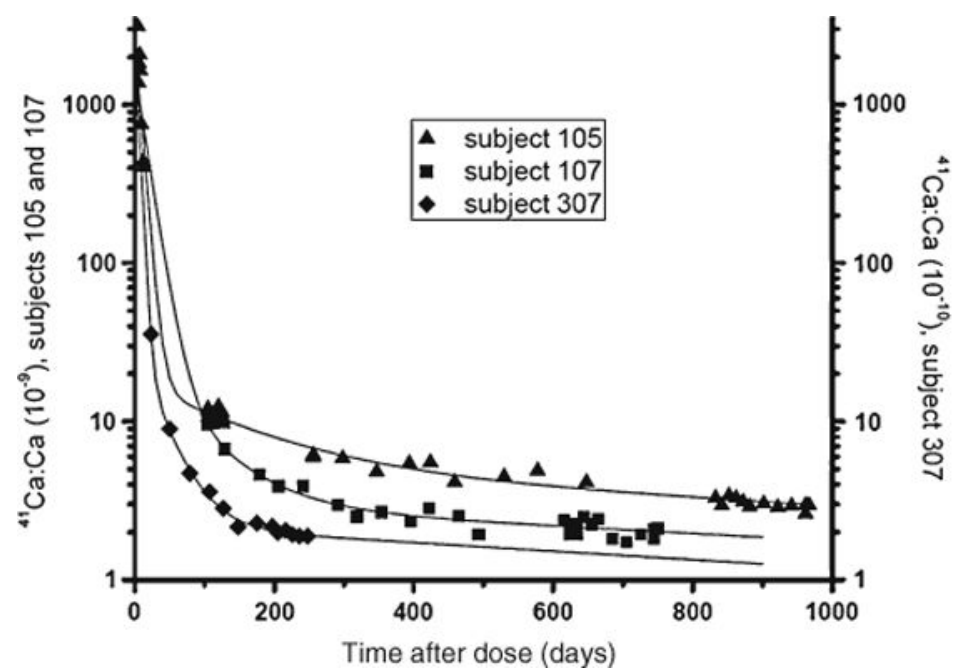

Fig. 2.

Calculated fits of the model (Fig. 1) (lines) to urine ${ }^{41} \mathrm{Ca}$ :Ca ratio data (symbols) for three subjects. Triangle and square symbols indicate two subjects who received $1 \mu \mathrm{Ci}$ (right $y$ axis, ${ }^{41} \mathrm{Ca}: \mathrm{Ca}$ ratio $\times 10^{-9}$ ), while diamond symbol indicates a subject who received $50 \mathrm{nCi}$ (left $y$-axis, ${ }^{41} \mathrm{Ca}: \mathrm{Ca}$ ratio $\times 10^{-10}$ ) 


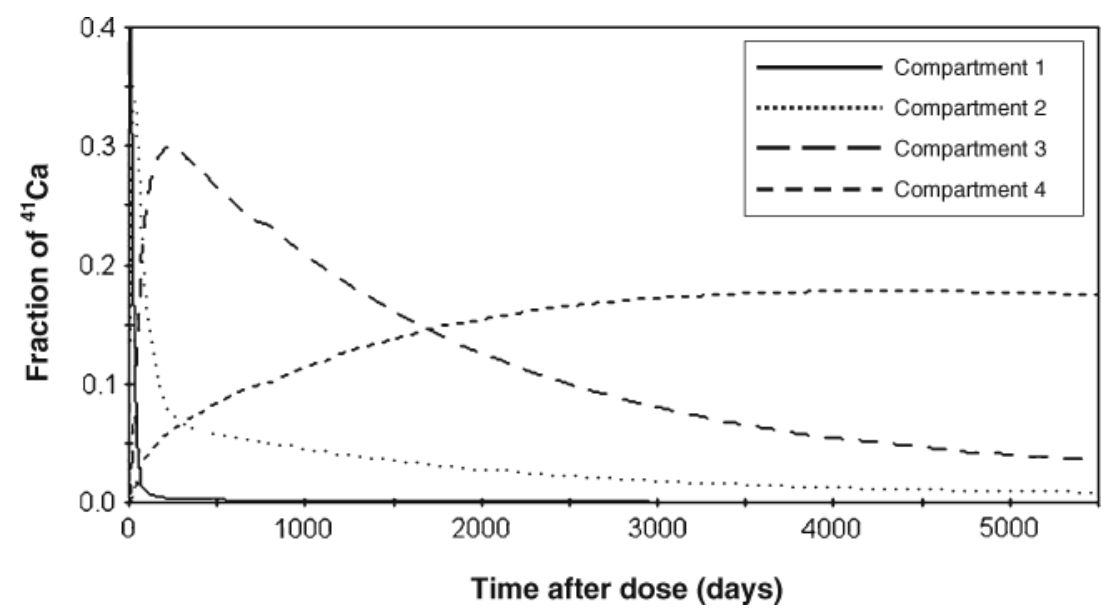

Fig. 3.

Simulation of distribution of the initial dose (fraction) between pools for the model in Fig. 1 over 5,000 days. Compartment 4 has the slowest rate of turnover, which is consistent with "cortical" bone, suggesting that interventions at 250 days post-dose mainly affect tracer in "trabecular" bone 


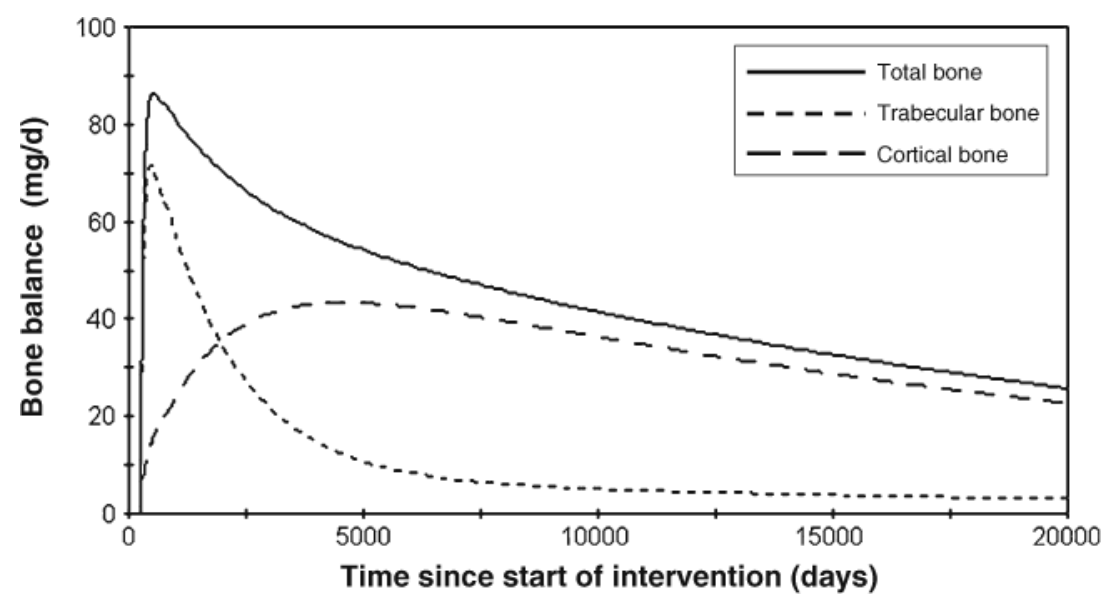

Fig. 4.

Simulation of bone balance (the sum of "trabecular" and "cortical" bone balances) over time with a sustained intervention (increased calcium absorption, urine excretion, and "trabecular" bone deposition, simulation 8 in Table 3). For this case, the simulation predicts that total bone balance gain persists. It takes many years to return to the pre-intervention, or zero state 


\section{Table 1}

Subject characteristics of post-menopausal women

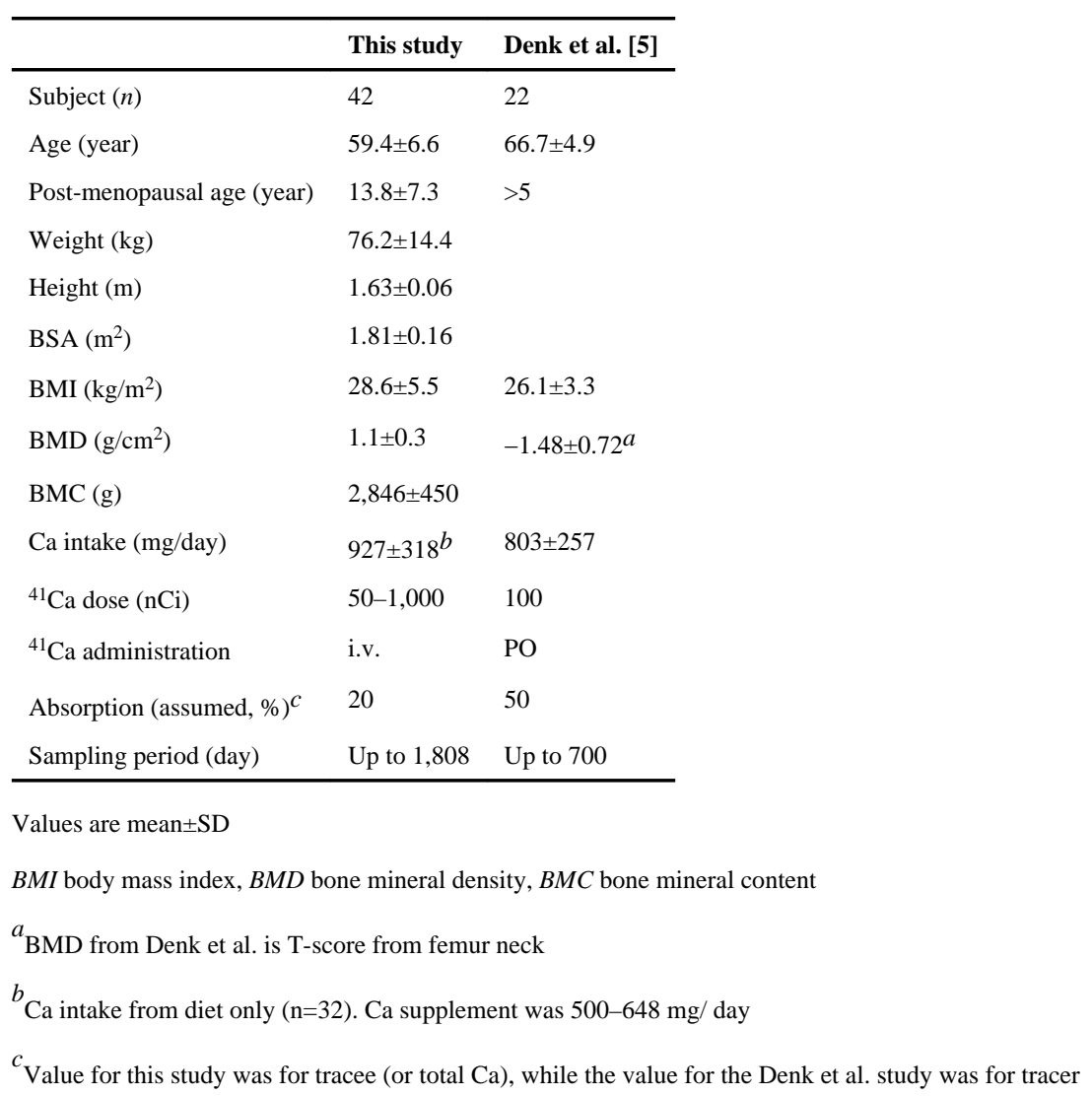




\section{Table 2}

Comparison of ${ }^{41} \mathrm{Ca}$ and ${ }^{45} \mathrm{Ca}$ kinetic study design and modeling results of bone resorption and urine calcium excretion using shared subjects $(n=13)$ in both studies

\begin{tabular}{|c|c|c|}
\hline & This study & Spence et al. [14] \\
\hline \multicolumn{3}{|l|}{ Study design } \\
\hline Tracer & ${ }^{41} \mathrm{Ca}$ & ${ }^{45} \mathrm{Ca}$ \\
\hline Dosing scheme & i.v. & Oral and i.v. \\
\hline Dosing amount & $50-1,000 \mathrm{nCi}$ & $10 \mu \mathrm{Ci}$ \\
\hline Sampling scheme & Urine & Blood, urine, and feces \\
\hline Sampling period (day) & Up to 1,808 & Up to 24 \\
\hline \multicolumn{3}{|l|}{ Calculated rates $^{a}$} \\
\hline Bone resorption (mg Ca/day) $b$ & $464 \pm 131$ & $388 \pm 125$ \\
\hline Urine excretion (mg Ca/day) $b$ & $149 \pm 131$ & $162 \pm 50$ \\
\hline
\end{tabular}

${ }^{a}$ Values are mean $\pm \mathrm{SD}$

${ }^{b}$ Values are not different between studies when using paired $t$ test $(p>0.05)$ 
Lee et al.

Page 19

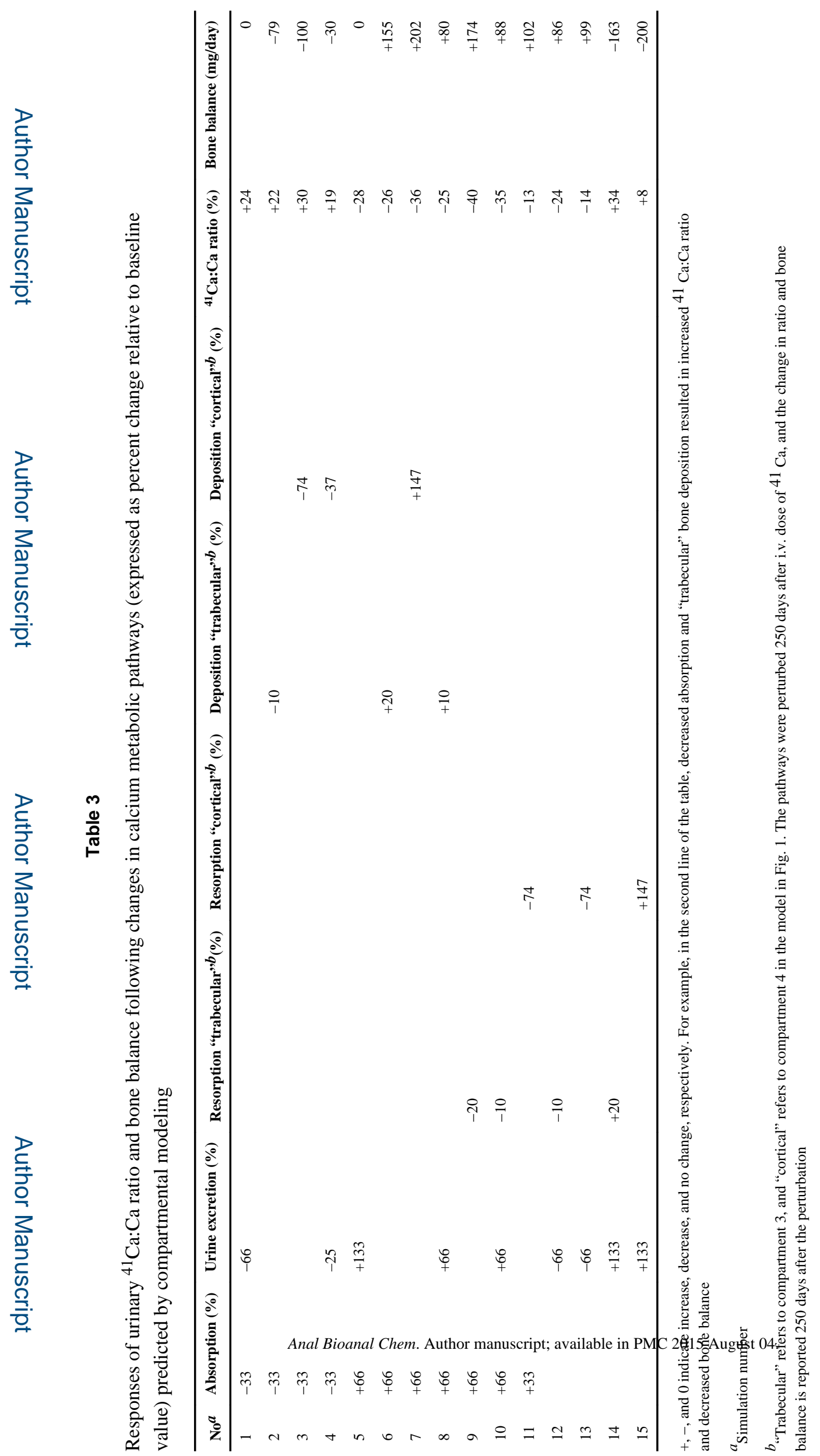


Lee et al.

Page 20

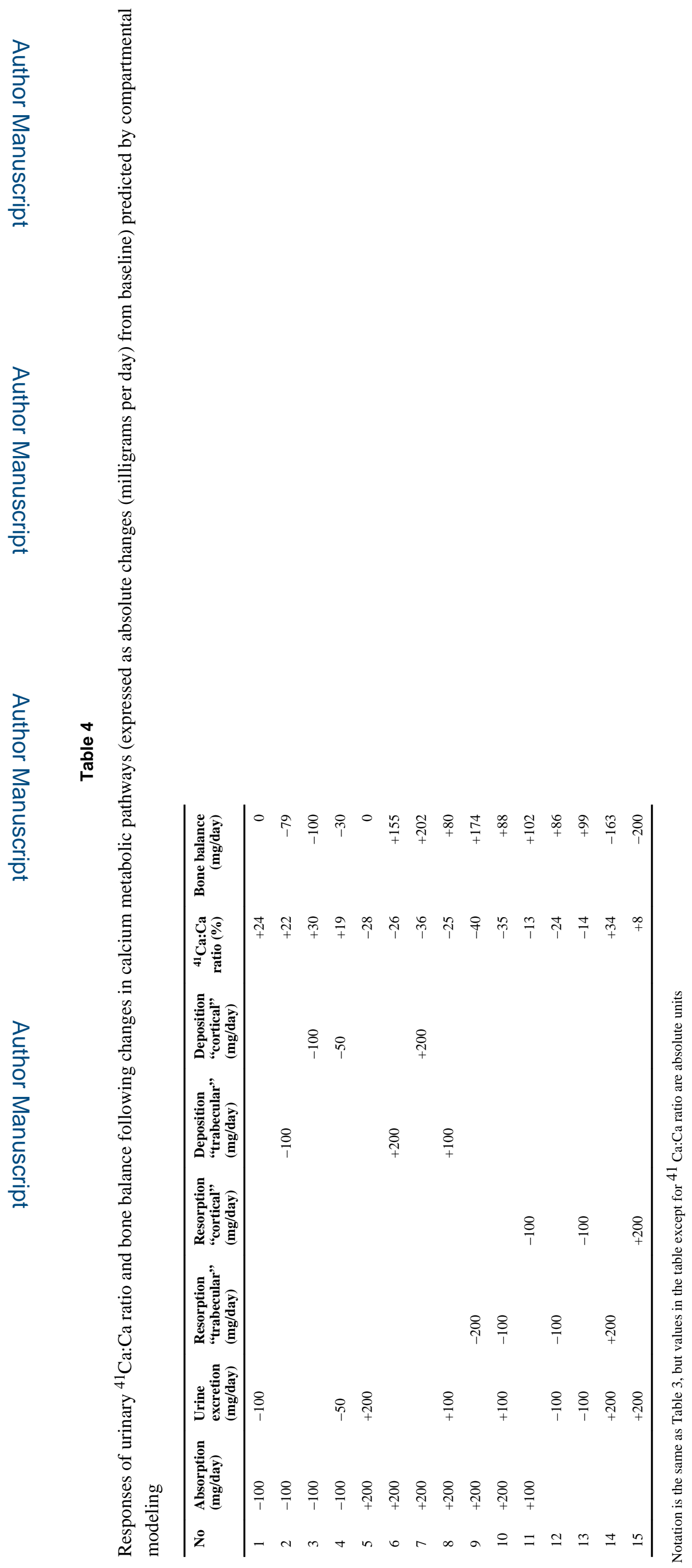

Anal Bioanal Chem. Author manuscript; available in PMC 2015 August 04. 
Table 5

Model simulation results comparing the time of introducing an intervention (250 vs. 2,000 days post-dose) on the change in urinary ${ }^{41} \mathrm{Ca}: \mathrm{Ca}$ ratio and bone balance caused by the intervention

\begin{tabular}{lccccc}
\hline \multirow{2}{*}{ Simulation no $^{a}{ }^{a}$} & \multicolumn{2}{c}{ Ratio (\%) } & & \multicolumn{2}{c}{ Bone balance (mg/day) } \\
\cline { 2 - 3 } \cline { 5 - 6 } & $\mathbf{2 5 0}$ days & $\mathbf{2 , 0 0 0}$ days & & $\mathbf{2 5 0}$ days & $\mathbf{2 , 0 0 0}$ days \\
\hline 6 & -26 & -18 & & 155 & 158 \\
7 & -36 & -40 & & 202 & 199 \\
12 & -24 & -21 & & 86 & 80 \\
13 & -14 & -16 & & 99 & 99 \\
\hline
\end{tabular}

Value indicates change from baseline value

${ }^{a}$ Simulation numbers match those in Table 3 